\title{
Radiation Damage in Electronic Memory Devices
}

\author{
Irfan Fetahović, ${ }^{1}$ Milić Pejović, ${ }^{2}$ and Miloš Vujisićc ${ }^{3}$ \\ ${ }^{1}$ State University of Novi Pazar, 36300 Novi Pazar, Serbia \\ ${ }^{2}$ Faculty of Electronic Engineering, University of Niš, 18000 Niš, Serbia \\ ${ }^{3}$ Faculty of Electrical Engineering, University of Belgrade, 11120 Belgrade, Serbia \\ Correspondence should be addressed to Irfan Fetahović; irfanfetahovic@gmail.com
}

Received 10 April 2013; Accepted 25 May 2013

Academic Editor: Predrag Osmokrovic

Copyright (c) 2013 Irfan Fetahović et al. This is an open access article distributed under the Creative Commons Attribution License, which permits unrestricted use, distribution, and reproduction in any medium, provided the original work is properly cited.

\begin{abstract}
This paper investigates the behavior of semiconductor memories exposed to radiation in order to establish their applicability in a radiation environment. The experimental procedure has been used to test radiation hardness of commercial semiconductor memories. Different types of memory chips have been exposed to indirect ionizing radiation by changing radiation dose intensity. The effect of direct ionizing radiation on semiconductor memory behavior has been analyzed by using Monte Carlo simulation method. Obtained results show that gamma radiation causes decrease in threshold voltage, being proportional to the absorbed dose of radiation. Monte Carlo simulations of radiation interaction with material proved to be significant and can be a good estimation tool in probing semiconductor memory behavior in radiation environment.
\end{abstract}

\section{Introduction}

All devices used by mankind are constantly exposed to different kinds of radiation originating from natural sources, but also from artificial manmade sources. Increasing the level of integration of electronic components and miniaturization trends may have negative influence onto the component sensitivity to ionizing radiation. Radiation defects can also occur in a process of a very large scale integrated circuits fabrica tion, since the process often includes bombardment with high-energy ions and photons.

The aim of this paper is to examine the behavior of semiconductor memories exposed to radiation in order to establish their applicability in a radiation environment. The experimental procedure has been used to test radiation hardness of commercial semiconductor memories. Different types of memory chips have been exposed to indirect ionizing radiation by changing radiation dose intensity. The effect of direct ionizing radiation on semiconductor memory behavior has been analyzed by using Monte Carlo simulation method.

\section{Radiation Effects in Basic Semiconductor Electronic Devices}

Changes and disruptions of irradiated memory characteristics depend on type of radiation, speed of energy deposition in semiconductor device, and the type of material the semiconductor structure is made of. Radiation effects can be divided into transient and permanent, depending on time an irradiated device needs to recover from radiation and restore its functionality [1-4].

False signals and false logic states in digital electronic circuits are transient radiation effects. These effects do not cause destructive changes; they often disappear after a short period of time or can be removed by applying simple measures. Therefore, transient effects can induce a type of errors named after soft errors. The examples of transient effects are disruptions caused by high radiation dose (doserate effects) or by heavy charged particle passing through a component (single event upsets-SEU) [5, 6]. Permanent radiation effect, or hard error, is a change in semiconductor 
structure after its component is being irradiated and cannot be easily resolved. Sometimes, these errors can be reversible after a long period of time. The examples of hard errors are latchup and snapback; they cannot be resolved without stopping the component $[4,5]$. The component destruction is hard error because impaired functionality cannot be restored.

\section{Radiation Damages in Semiconductor Memories}

Semiconductor memories are often a limiting factor in applying a device in a radiation environment since they are most prone to damage when exposed to radiation, comparing to other semiconductor integrated circuits $[5,6]$.

Memory errors can occur under influence of a cosmic radiation or alpha particles emitted from memory chip package. Thorium and uranium isotopes' radiation, as well as radiation of alpha emitters, can lead to soft error occurrences in memories. These errors are often manifested as logic state disturbances of memory cells or disturbances in sense amplifiers; it is seldom a function disturbance of peripheral circuits in a memory chip. In cosmic applications, light and heavy ions can influence the semiconductor memories' characteristics, causing both soft and hard errors.

In different parts of memory chip, radiation can induce sudden occurrence of charged carriers-electrons and holes. These particles generate false currents that might disturb data written into the chip or influence data processing. These types of disturbances in memory are called soft errors, and when induced by radiation they are called transient upsets. Single event upsets (SEU), caused by heavy charged particle passing through memory, are very significant for radiation compatibility of semiconductor memories, since frequent occurrence of single event upsets has a negative impact on component's mean time to failure [6-8].

\section{The Experiment}

4.1. Gamma Radiation Experimental Procedure. Radiation effects on EPROM and EEPROM semiconductor memories have been tested by exposing samples to gamma radiation, the source of radiation being ${ }^{60} \mathrm{Co}$.

The used components have not been exposed to the effects of neutron radiation since they belong to the group of MOS structures, where the effects of neutron radiation are significantly less comparing to gamma radiation.

All memory locations of the samples have been initially written into logic " 1 " state, since this state is more radiation sensitive than the logic "0" state. Testing has been performed in steps, by increasing absorbed dose of radiation within each step. During irradiation, some memory cells changed its logic state from "1" to " 0 ," and the very process has been defined as an error. After irradiation, memory has been read, and the number of logic " 0 " state has been observed, being the same as the number of errors.

The experimental measurement results were collected with combined uncertainty lower than $10 \%[9,10]$.
4.2. Simulation of Direct Ionizing Radiation Interaction with Semiconductor Memory. Monte Carlo simulations of interaction between direct ionizing radiation (protons and alpha particles) and MOS structure insulator layer have been performed in TRIM software, being part of SRIM 2008 programming package. TRIM is as open source software made for simulation of ions travelling through the material [11]. It is based on using a quantum mechanical treatment of ion-atom collisions $[12,13]$.

\section{Results and Discussion}

5.1. Indirect Ionizing Radiation Damage. Figures 1 and 2 show the average relative change in number of errors versus the absorbed dose of radiation, in irradiated EPROM and EEPROM samples, respectively. The number of errors is proportional to the absorbed dose for both EPROM and EEPROM memory samples. The first error in EPROM samples has been observed within the absorbed dose at $1110 \mathrm{~Gy}$; the dose being greater than $1230 \mathrm{~Gy}$, the number of error has become significantly larger. EEPROM memories proved to be more sensitive on gamma radiation, since the first error has been observed within radiation dose of $880 \mathrm{~Gy}$.

In both EPROMs and EEPROMs, the floating gate is used to store charge and thus maintain a logical state. The stored charge determines the value of transistor threshold voltage, making the memory cell either "on" or "off" at readout.

Gamma radiation causes generation of electron-hole pairs in $\mathrm{SiO}_{2}$ insulator of the gate. The number of generated pairs is directly proportional to the energy deposited in material, depending on the total absorbed dose of radiation $[8,14]$. The part of generated pairs is recombined, depending on electric field strength. Being more mobile than the holes, electrons leave the insulator, influenced by the gate voltage. The generated holes slowly drift towards floating gate (FG). The fraction of holes becomes captured in trapping sites, forming positive radiation-induced charge in insulator. The remains of holes flow into the FG, reducing the number of electrons within it, thus influencing the logical state of a memory cell.

5.2. Direct Ionizing Radiation Damage. The calculation is made very efficient using statistical algorithms allowing the ion to make jumps between calculated collisions and then averaging the collision results over the intervening gap. The interaction of $50 \mathrm{keV}$ protons and $30 \mathrm{keV}$ alpha particles with $0.5 \mu \mathrm{m}$ thick $\mathrm{SiO}_{2}$ oxide layer has been simulated. The results are presented in Figures 3 and 4.

During inelastic scattering on electrons, the incident particle can transfer energy to the atom, raising it to a higher energy level (excitation), or it may transfer enough energy to remove an electron from the atom (ionization). A portion of energy transferred during each individual scattering of heavy charged particles on electrons is small compared to an entire kinetic energy of a particle, but the number of particle collisions per a unit path length is as high that the entire total energy loss is significant even in relatively thin material layers. 


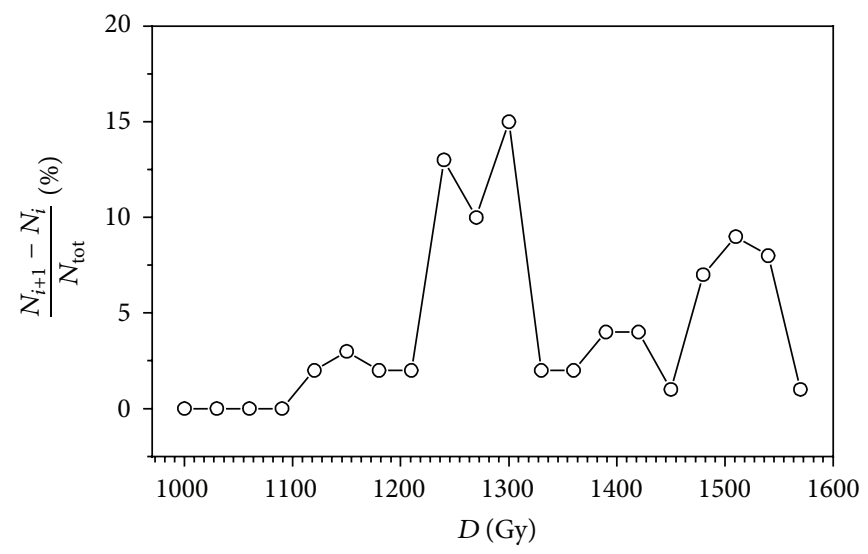

(a)

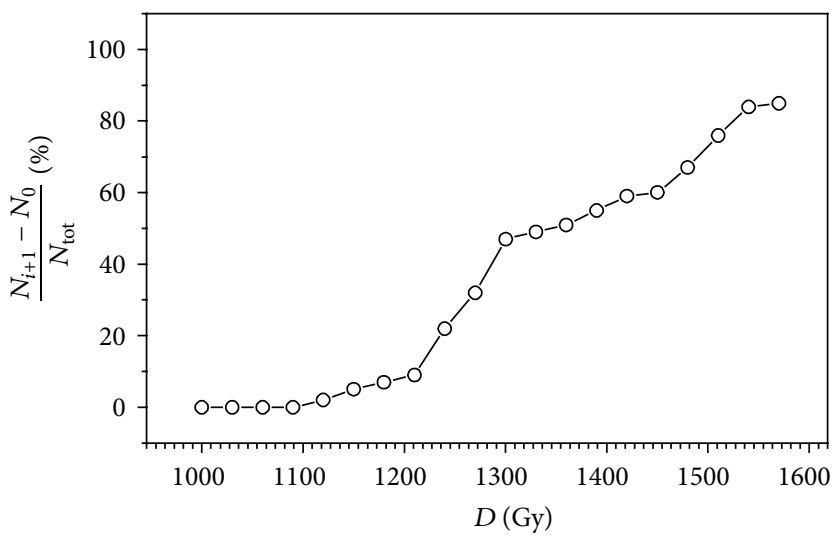

(b)

FIGURE 1: The average relative change in number of errors in irradiated EPROM samples (NM27C010) versus the absorbed dose of radiation: (a) differential; (b) cumulative $\left(N_{\text {tot }}=1,048,576\right.$ bit, $\left.N_{0}=0\right)$.

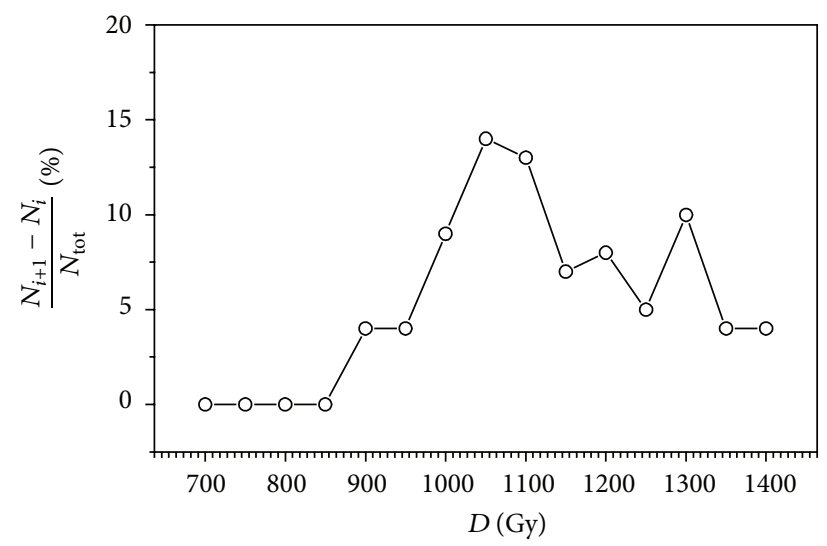

(a)

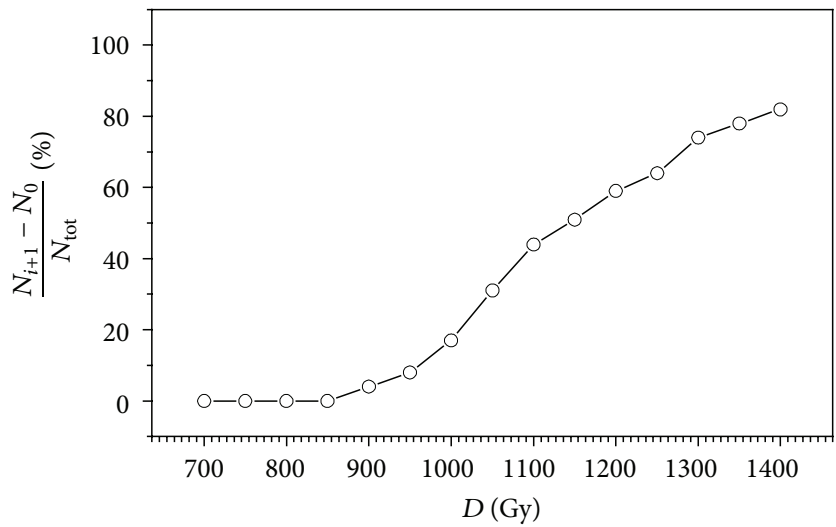

(b)

FIGURE 2: The average relative change in number of errors in irradiated EEPROM samples (NM93CS46) versus the absorbed dose of radiation: (a) differential; (b) cumulative $\left(N_{\text {tot }}=1024\right.$ bit, $\left.N_{0}=0\right)$.

The energy loss of heavy charged particles per a unit path length increases at the end of their path. At the very end of its path, when the particles have already lost the greatest part of their energy, the ones start to capture electrons, while a stopping power and specific ionization rapidly decrease [15]. Alpha particles are heavier than protons, and their energy is lower than the energy of protons, so a penetration depth of alpha particles in target $\mathrm{SiO}_{2}$ layer is lower compared to the penetration depth of protons, as indicated in Figures 3(a) and 4(a).

Elastic scatterings on the nuclei lead to atom ejections from their positions in a crystal lattice, producing displacement damages. There is a silicon nucleus displacement when the energy of an incident particle (projectile) transferred to the nucleus in the elastic collision is higher than a threshold displacement energy $E_{d}$. Such ejected silicon atom is called primary knock-on atom (PKA). An empty space in the crystal lattice produced by $\mathrm{Si}$ atom displacement is called a vacancy. When the displaced atom occupies a site among lattice nodes (interstitial site), the vacancy and the interstitial Si atom together represent Frenkel pair $\left(V+(\mathrm{Si})_{i}\right)[16]$. Kinetic energy of the PKA being equal to the difference of the energy transferred to the atom in the elastic collision and the threshold displacement energy can be large enough so as the atom, before occupying its interstitial site, causes displacement of the entire set of other nearby nuclei that can themselves displace other nuclei, producing a collision cascade. Along its path, the recoil atoms lose their energy in two ways-by ionization and displacement of other nuclei. Figure 4(b) contains two distinct plots, one for electronic energy loss from the incident alpha particles and one for energy loss from displaced atoms. The ionization energy loss is greater within alpha particles than within recoil atoms. The electrons tend to absorb energy most efficiently from particles whose velocity is similar to their velocity. The alpha particles lose more energy to the target electrons since they move much faster than the recoil atoms.

Point defects in an insulator, produced as a result of displacement damages, can serve as the trapping sites for charge carriers. These imperfections may alter material properties of 


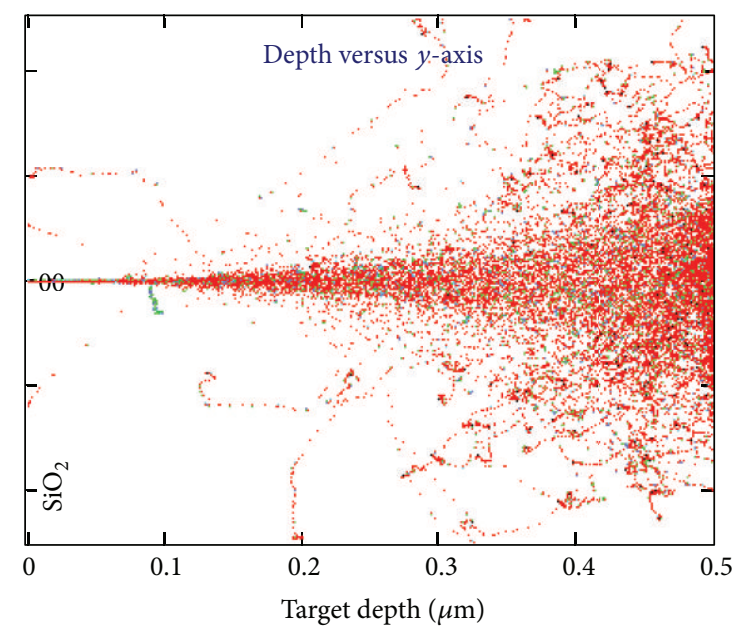

(a)

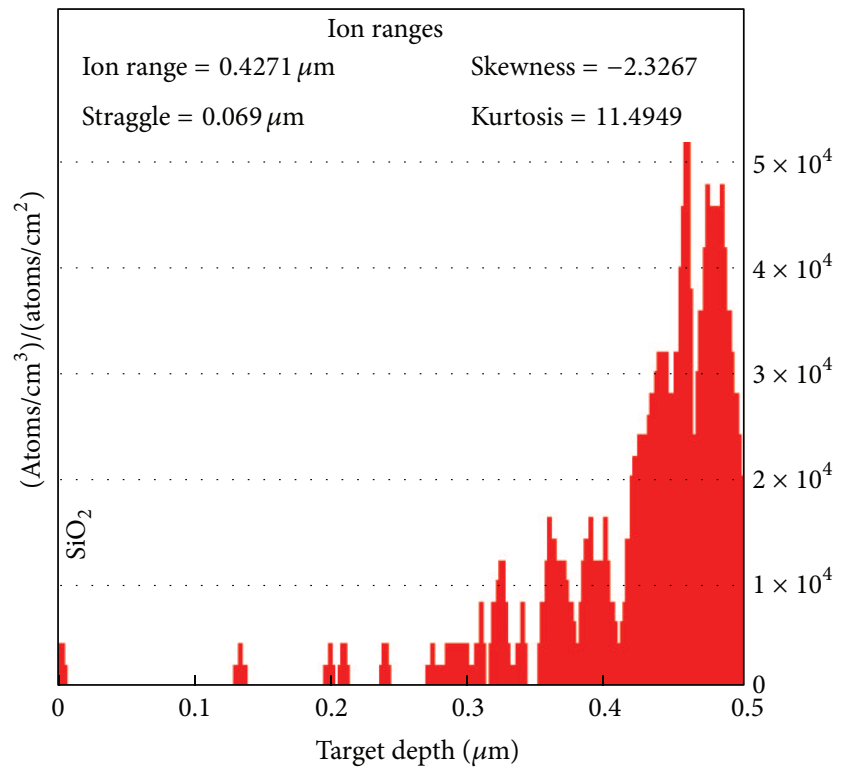

(b)

FIGURE 3: The simulation of $50 \mathrm{keV}$ protons passing through $0.5 \mu \mathrm{m}$ thick layer of $\mathrm{SiO}_{2}$ (500 events). (a) Trajectory of protons in $x \mathrm{O} y$ plane. (b) Distribution of stopped protons along the oxide depth.

the insulator, thus changing and disrupting characteristics of an electronic memory device which the insulator is part of [17].

\section{Conclusion}

Based on analysis of data gathered from performed experiments, the exposure of semiconductor memories to gamma radiation causes three effects: holes being captured in trapping sites of an oxide, injection of holes from oxide into FG, and emission of electrons through FG-oxide interface.

The generation of electron-hole pairs leads to trapping of positive-charged carriers (holes) in insulator, causing negative shift in $I_{D} / V_{G}$ characteristics. Namely, positive-charged

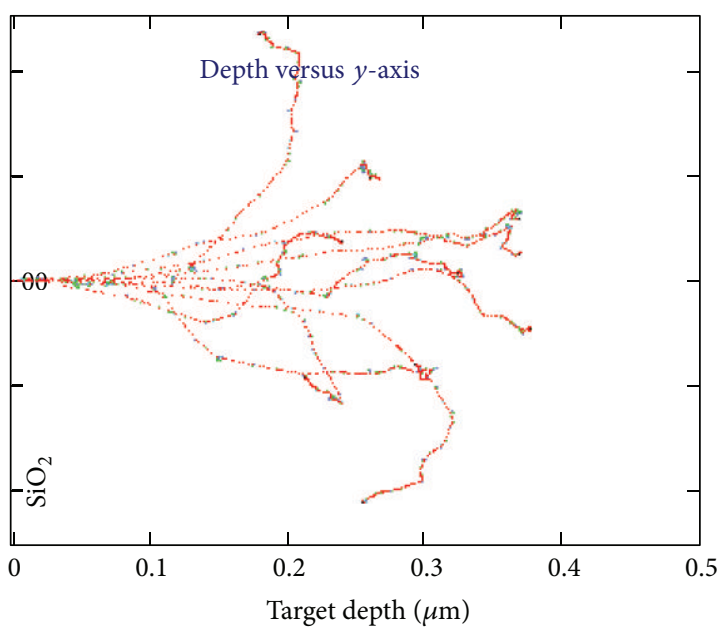

(a)

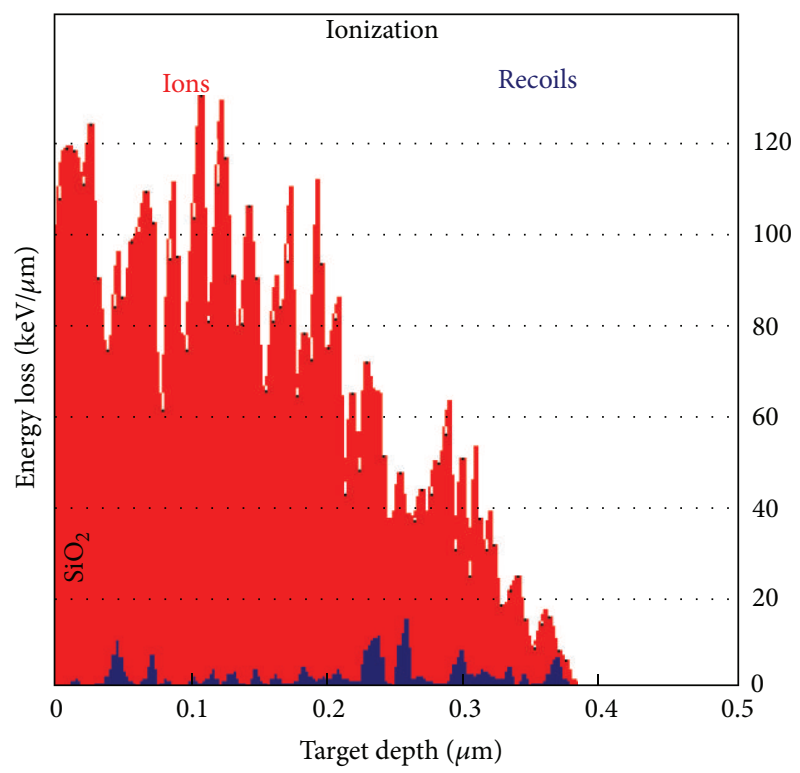

(b)

FIgURE 4: The simulation of $30 \mathrm{keV}$ alpha particles passing through $0.5 \mu \mathrm{m}$ thick layer of $\mathrm{SiO}_{2}$ (10 events). (a) Trajectory of alpha particles in $x \mathrm{O} y$ plane. (b) The ratio of specific ionization (linear transfer of energy) of incident alpha particles and displaced $\mathrm{Si}$ and $\mathrm{O}$ atoms along the oxide depth.

carriers induced by gamma radiation require the increase of negative gate voltage to compensate the positive charge. It means that gamma radiation causes decrease in threshold voltage, being proportional to the absorbed dose of radiation. The future research of radiation compatibility of semiconductor memories can be directed towards analyzing the influence of gate oxide material and oxide thickness and examining the effects of absorbed dose rate. Monte Carlo simulations of radiation interaction with material proved to be significant and can be a good estimation tool in probing semiconductor memory behavior in radiation environment. 


\section{Acknowledgment}

The Ministry of Education, Science and Technological Development of the Republic of Serbia supported this work under Contract no. 171007.

\section{References}

[1] J. L. Barth, C. S. Dyer, and E. G. Stassinopoulos, "Space, atmospheric, and terrestrial radiation environments," IEEE Transactions on Nuclear Science, vol. 50, no. 3, pp. 466-482, 2003.

[2] V. S. Vavilov and N. A. Ukhin, Radiation Effects in Semiconductors and Semiconductor Devices, Consultants Bureau, New York, NY, USA, 1977.

[3] R. Radosavljević and A. Vasić, "Effects of radiation on solar cells as photovoltaic generators," Nuclear Technology \& Radiation Protection, vol. 27, no. 1, pp. 28-32, 2012.

[4] D. Nikolić, A. Vasić, I. Fetahović, K. Stanković, and P. Osmokrović, "Photodiode behavior in radiation environment," Scientific Publications of the State University of Novi Pazar A, vol. 3, no. 1, pp. 27-34, 2011.

[5] G. Holmes-Siedle and L. Adams, Handbook of Radiation Effects, Oxford University Press., Oxford, UK, 2nd edition, 2002.

[6] G. C. Messenger and M. S. Ash, The Effects of Radiation on Electronic Systems, Van Nostrand Reinhold, New York, NY, USA, 1992.

[7] M. Vujisić, N. Marjanović, I. Fetahović, K. Stanković, and P. Osmokrović, "Influence of radiation on titanium dioxide mermistors," Scientific Publications of the State University of Novi Pazar A, no. 1, pp. 75-82, 2012.

[8] M. Zdravković, A. Vasić, R. Radosavljević, M. Vujisić, and P. Osmokrović, "Influence of radiation on the properties of solar cells," Nuclear Technology \& Radiation Protection, vol. 26, no. 2, pp. 158-163, 2011.

[9] K. Stanković, "Influence of the plain-parallel electrode surface dimensions on the type A measurement uncertainty of GM counter," Nuclear Technology \& Radiation Protection, vol. 26, no. 1, pp. 39-44, 2011.

[10] Ć. Dolićanin, K. Stanković, D. Dolićanin, and B. Lončar, "Statistisal treatment of nuclear counting results," Nuclear Technology \& Radiation Protection, vol. 26, no. 2, pp. 164-170, 2011.

[11] J. F. Ziegler, J. P. Biersack, and M. D. Ziegler, "SRIM (The Stopping and Range of Ions in Matter)," http://www.srim.org.

[12] J. H. Warner, S. R. Messenger, R. J. Walters, and G. P. Summers, "Displacement damage correlation of proton and silicon ion radiation in GaAs," IEEE Transactions on Nuclear Science, vol. 52, no. 6, pp. 2678-2682, 2005.

[13] M. Vujisic, K. Stankovic, N. Marjanovic, and P. Osmokrovic, "Simulated effects of proton and ion beam irradiation on titanium dioxide memristors," IEEE Transactions on Nuclear Science, vol. 57, no. 4, pp. 1798-1804, 2010.

[14] S. Stanković, B. Iričanin, D. Nikolić et al., "MSV signal processing systemfor neutron-gamma discrimination in a mixed field," Nuclear Technology \& Radiation Protection, vol. 27, no. 2, pp. 165-170, 2012.

[15] J. R. Srour, Basic Mechanisms of Radiation Effects on Electronic Materials, Devices and Integrated Circuits, DNA-TR-82-20, New York, NY, USA, 1982.

[16] J. R. Schwank, M. R. Shaneyfelt, D. M. Fleetwood et al., "Radiation effects in MOS oxides," IEEE Transactions on Nuclear Science, vol. 55, no. 4, pp. 1833-1853, 2008.
[17] G. Cellere, A. Paccagnella, A. Visconti, and M. Bonanomi, "Ionizing radiation effects on floating gates," Applied Physics Letters, vol. 85, no. 3,3 pages, 2004. 

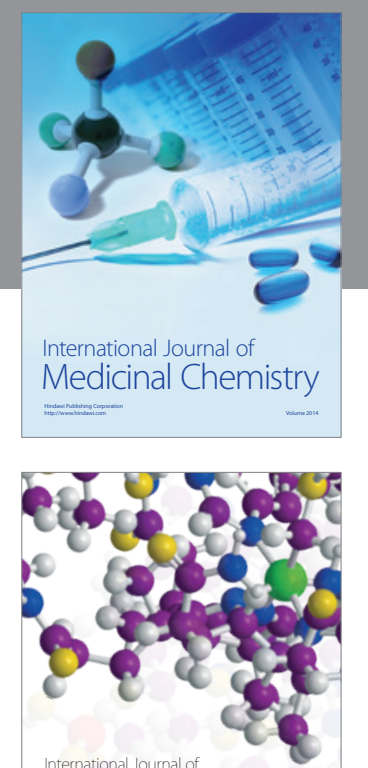

\section{Carbohydrate} Chemistry

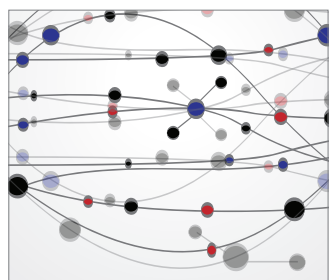

The Scientific World Journal
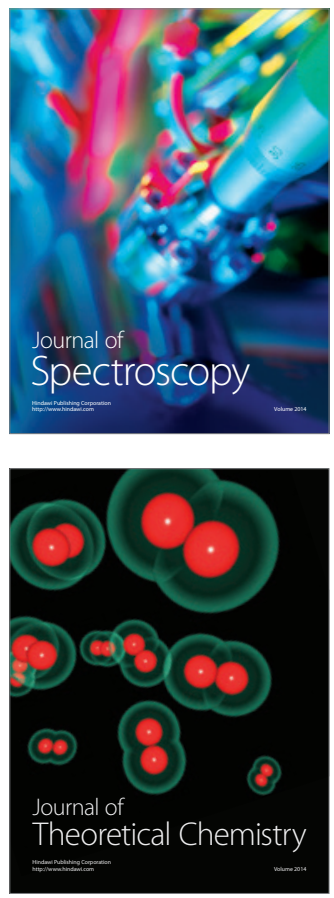
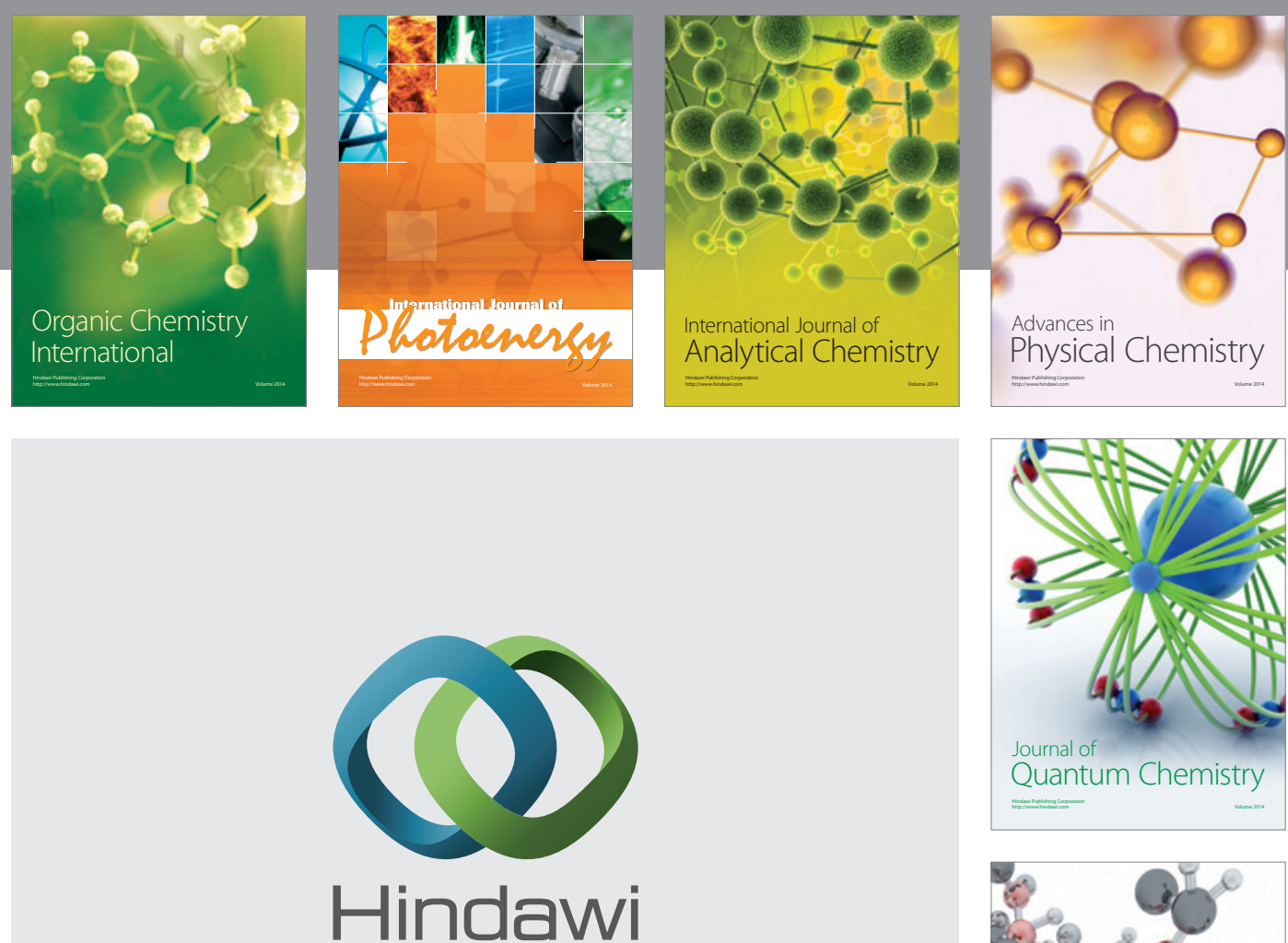

Submit your manuscripts at

http://www.hindawi.com

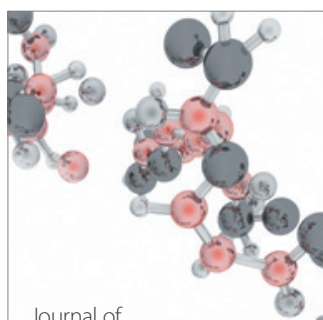

Analytical Methods

in Chemistry

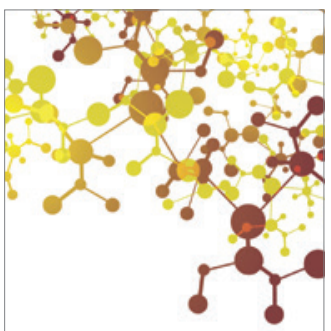

Journal of

Applied Chemistry

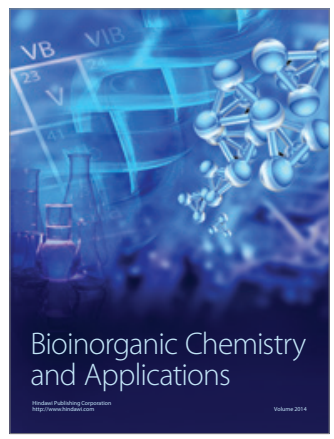

Inorganic Chemistry
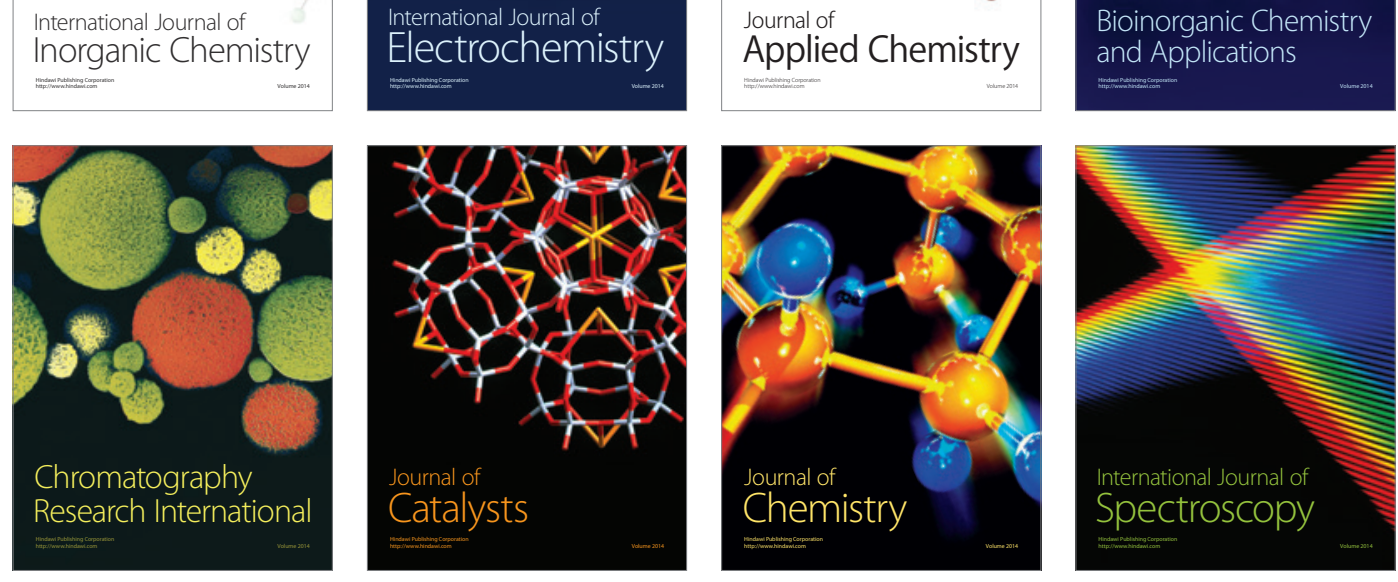